\title{
NK cell effector functions in a Chédiak-Higashi patient undergoing cord blood transplantation: Effects of in vitro treatment with IL-2
}

\author{
Loredana Cifaldi $^{\mathrm{a}, *}$, Rita Maria Pinto ${ }^{\mathrm{a}}$, Ippolita Rana ${ }^{\mathrm{a}}$, Maurizio Caniglia ${ }^{\mathrm{a}, \mathrm{b}}$, \\ Adriano Angioni ${ }^{c}$, Stefano Petrocchi ${ }^{c}$, Caterina Cancrini ${ }^{\mathrm{d}}$, Laura Cursi ${ }^{\mathrm{a}}$, \\ Giuseppe Palumbo ${ }^{\mathrm{d}}$, Alessandra Zingoni ${ }^{\mathrm{e}}$, Angela Gismondi ${ }^{\mathrm{e}}$, Paolo Rossi ${ }^{\mathrm{d}}$, \\ Angela Santoni ${ }^{\mathrm{e}}$, Cristina Cerboni ${ }^{\mathrm{e}}$ \\ a Department of Pediatric Hematology and Oncology, Bambino Gesù Children's Hospital, IRCCS, Rome, Italy

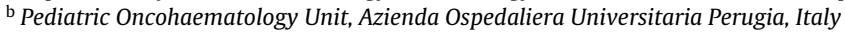 \\ c Cytogenetics and Molecular Genetics Unit, Bambino Gesù Children's Hospital, IRCCS, Rome, Italy \\ d University Department of Pediatrics, Bambino Gesù Children's Hospital and University of Tor Vergata, School of Medicine, Rome, Italy \\ e Department of Molecular Medicine, Istituto Pasteur-Fondazione Cenci Bolognetti, "Sapienza" University of Rome, Italy
}

\section{A R T I C L E I N F O}

\section{Article history:}

Received 17 October 2016

Received in revised form 27 October 2016

Accepted 28 October 2016

Available online 2 November 2016

\section{Keywords:}

NK cells

Immunodeficiency

Cellular immunology

Interleukin 2

Cord blood transplantation

\begin{abstract}
A B S T R A C T
NK cell cytotoxicity in Chédiak-Higashi syndrome (CHS) is strongly impaired as lytic granules are not released upon NK-target cell contact, contributing to several defects typical of this severe immunodeficiency. Correction of NK cell defects in CHS should improve the outcome of hematopoietic stem-cell transplantation, proposed as therapy.

We investigated NK cell functions in a CHS patient before and after cord-blood transplantation, and the ability of in vitro IL-2 treatment to restore them.

Before the transplant, the strong defect in NK cell-mediated natural and antibody-dependent cytotoxicity, as well as in IFN- $\gamma$ production, could be restored up to normal levels by in vitro IL-2 treatment. This cytokine also caused the appearance of smaller lysosomal granules and their orientation towards the NK-target cell contact area, thus suggesting that IL-2 had a more general capacity to restore NK cell effector functions. Moreover after the transplant, although the successful engraftment, NK cell cytotoxicity resulted still partially impaired at one year, almost normal at ten years and, anyhow, fully recovered by in vitro IL-2 treatment.

Taken together, our results indicate that IL-2 had a wide capacity to restore NK cell effector functions, being able to reverse the altered cytotoxic activity, lytic granule pattern, and cytokine production observed in the CHS patient.
\end{abstract}

(C) 2016 Published by Elsevier B.V. on behalf of European Federation of Immunological Societies

\section{Introduction}

The Chédiak-Higashi syndrome (CHS) is a rare childhood autosomal recessive disease [1] characterized by LYST gene alterations, described so far as nonsense or frameshift mutations and resulting in a truncated protein [2]. The impaired protein function, leads to the perinuclear concentration of giant granules in circulating cyto-

Abbreviations: CHS, Chédiak-Higashi Sindrome; NK cells, Natural Killer cells; AP, Accelerated Phase; IL-2, interleukin 2; BMT, bone-marrow transplantation; UCB-T, umbilical-cord blood transplantation.

* Corresponding authors at: Department of Pediatric Hematology and Oncology, Bambino Gesù Children's Hospital, Viale di San Paolo 15, 00146 Rome, Italy.

E-mail address: loredana.cifaldi@opbg.net (L. Cifaldi). toxic lymphocytes and in many other cell types, the presence of which represents the hallmark of CHS. As reported recently, missense mutations in the different LYST domains lead to a different size and immunological synapse distribution of the lysosome granules, thus suggesting that a specific LYST mutation could differently affect disease phenotype [3].

Defective phagocyte, T lymphocyte and NK cell functions, including both natural and antibody-dependent cell cytotoxicity (ADCC), are likely to contribute to the enhanced susceptibility to infections [4-8]. The onset of the so-called "accelerated phase" (AP), a pathological condition mainly characterized by a critical impairment of the immune system [9], together with the patient age, have been reported as key prognostic factors of CHS [1]. Most of the patients die young because of AP, which occurs in the majority of individuals (85\%) any time from the first 
months after birth, to several years later. Most of the patients with AP suffer a hemophagocytic-syndrome-like lymphoproliferative disorder, associated with fever, episodes of abnormal bleeding, overwhelming infections and organ failure. Although AP is cured with etoposide combined with steroids and intrathecal methotrexate, remission is transient, relapses become insensitive to treatment and patients die before the age of ten years because of bleeding complications and infections $[1,9]$.

For this reason, at present, the most significant clinical intervention consists in hematopoietic stem cell (HSC) transplantation, mainly of bone marrow (BM) origin [10]. However, the use of umbilical-cord blood transplantation (UCB-T) in patients with CHS has been recently reported [11].

Lack of NK cell functions in CHS patients has been associated to the increased susceptibility to viral infections, and has been theorized to allow AP to become fulminant [1,9]. Restoration of NK cell activities, and consequently, of innate immunity against viruses, might thus represent a therapeutic strategy for these patients. Here we show the potentiality of IL-2, a master cytokine promoting NK cell proliferation and effector functions, in restoring in vitro the NK cell functional defects of a 2 month-old child affected by CHS, initially undergoing anti-blastic therapy and then UCB-T. NK cells isolated from the patient peripheral blood at the time of diagnosis, showed a severe impairment of their effector functions, which were however completely restored by in vitro IL-2 treatment. Similarly, after the successful UCB-T, we observed in ten-year follow-up an improvement of NK cell-mediated cytotoxicity upon IL-2 treatment. The restoration of NK cell functions mediated by i) UCB transplantation and ii) IL-2 treatment, might thus represent an important clinical intervention to improve the benefit of standard therapies for CHS patients.

\section{Materials and methods}

\subsection{CHS patient and pediatric healthy donors}

We describe a case of a 2-month old Caucasian male newborn of consanguineous parents, admitted to our hospital (March 2004) with fever, anemia, thrombocytopenia, neutropenia, hepatosplenomegaly, and negative virological tests (cytomegalovirus, EBV, HIV, HCV, HBV, toxoplasma). The occurrence of macula and hair hypopigmentation, the presence of giant granules in $\mathrm{BM}$ myeloid precursors (Fig. 1A) and of activated peripheral blood $\mathrm{CD}^{+}$ T lymphocytes $\left(65 \%\right.$ were HLA-DR ${ }^{+}$), helped us for the diagnosis of CHS. Genetic analysis, conducted at the Hôpital Necker-Enfants Malades (INSERM U429 Paris), revealed a homozygous region for the polymorphic markers linked to the LYST locus.

During hospitalization, the patient presented AP with the following symptoms and signs: persistent fever, opisthotonous, neck rigor, hepato-splenomegaly, oxygen desaturation, pancytopenia, hypertriglyceridaemia, hypofibrinogenaemia and hyposodiaemia. Anti-blastic therapy with etoposide, intrathecal methotrexate (MTX) and dexamethasone was started, with a mild improvement of clinical conditions. Even if the child was not in complete remission of AP (May 2004), after the identification of a UCB unit HLA $5 / 6$ matched (with a DRB1 locus difference), informed consent from the parents was obtained and allogeneic stem cell transplant was performed. The patient received a conditioning regimen based on etoposide $\left(150 \mathrm{mg} / \mathrm{m}^{2}, 3\right.$ days), busulfan $(5 \mathrm{mg} / \mathrm{kg} /$ day, 4 days), cyclophosphamide ( $50 \mathrm{mg} / \mathrm{kg} /$ day, 4 days). Graft versus host disease (GVHD) prophylaxis consisted of cyclosporine (CSA) ( $1 \mathrm{mg} / \mathrm{kg} /$ day from day -7 to day -2 , then $3 \mathrm{mg} / \mathrm{kg} /$ day until day $90)$, and methylprednisolone ( $1 \mathrm{mg} / \mathrm{kg}$ until day +30 , then tapered). Engraftment was achieved at day +10 . Fifteen days after UCB-T, the patient presented a severe (grade IV) acute GVHD with skin, hepatic and gut involvement, treated with steroids ( $5 \mathrm{mg} / \mathrm{kg} /$ day for 7 days, then $2 \mathrm{mg} / \mathrm{kg}$ ) and CSA ( $3 \mathrm{mg} / \mathrm{kg} / \mathrm{day})$, and in absence of a good response, with 4 doses of anti-TNF- $\alpha$ monoclonal antibody (Remicade, $10 \mathrm{mg} / \mathrm{kg}$ once a week) and association of mycophenolate mofetil (30 mg/kg/day) with a clinical progressive improvement. The patient presented also a severe venocclusive disease treated with defibrotide $(60 \mathrm{mg} / \mathrm{kg} / \mathrm{day})$, CMV infection treated with ganciclovir, adenovirus infection treated with cidofovir. Six months after UCB-T, the patient was in good clinical conditions with no signs of AP, and a complete GVHD and viral infection control. Full donor leukocyte engraftment was confirmed by subsequent serial short tandem repeats (STR) determinations (December 2004), performed also on selected leukocyte subsets (March 2014) as T, NK, B cells, and monocytes, pre-isolated by cell-sorting (FACS Aria, Becton Dickinson). At 10 years of age, the patient kept good clinical conditions, despite showing some of the classical signs of the syndrome, such as albinism and silver-grey hair. Informed consent from parents of the patient as well as the prior approval from our institutional research ethics committee to conduct this study was obtained.

Pediatric healthy donors were children admitted to our hospital for routine health checkup and exhibiting normal complete WBC counts.

\subsection{Reagents}

The following monoclonal antibodies (mAbs) from Becton Dickinson (San Diego, CA) were used: control $\operatorname{IgG}_{1}$-FITC and -PE, anti-CD16 + anti-CD56-PE/anti-CD3-FITC, anti-CD3-FITC, antiCD56-PE, anti-CD8-Cy5, anti-IFN- $\gamma$ - FITC, anti-HLA-DR, anti-2B4 anti-NKp44. Other antibodies were: FITC-conjugated anti-perforin mAb (Ancell, Bayport, MN), anti-NKG2D (MAB149810) (R\&D Systems, Minneapolis, MN); anti-CD16 (for reverse ADCC; clone B73.1 kindly provided by Giorgio Trinchieri; National Cancer Institute, MD); anti-CD94/NKG2A (clone Z199) (Immunotech, Marseille, France); anti-LFA-1/beta2 integrin TS1/18 (Dr. F. Sanchez-Madrid, La Princesa Hospital, University of Madrid, Spain); F(ab') ${ }_{2}$ fragments of PE-conjugated goat-anti-mouse (GAM-PE) IgG (Jackson Immunoresearch Laboratories, Newmarket, UK). Other reagents were: recombinant human IL-2 (Peprotech; Rocky Hill, NJ); brefeldin A (Sigma) and rhodamine phalloidin ( $3 \mu \mathrm{M}$; Molecular Probes).

\subsection{Human NK cell preparation}

Polyclonal NK cell cultures were obtained by co-culturing peripheral blood mononuclear cells (PBMCs) $\left(4 \times 10^{5}\right.$ cells) isolated from the patient at different treatment time points (before the antiblastic therapy, upon drug treatment before UCB transplantation, 7 months after UCB transplantation, 13 months after UCB transplantation, 10 years after UCB transplantation), or from healthy donors, with the irradiated (30 Gy) EBV-transformed Bcell line RPMI8866 $\left(1 \times 10^{5}\right.$ cells) for $10-13$ days, as previously described $[12,13]$. These cells were used as effectors in cytotoxicity assays. PBMC (on day 0) and NK cell cultures (on day 10-13) were routinely checked for the percentage of $\mathrm{CD} 56^{+} \mathrm{CD} 16^{+} \mathrm{CD} 3^{-}$by FACS analysis. In some experiments, NK cells were stimulated with IL-2 $(250 \mathrm{IU} / \mathrm{ml})$ for $18 \mathrm{~h}$ at $37^{\circ} \mathrm{C}$.

\subsection{Cytotoxicity assays}

K562, a human erythroleukemia cell line, was the target for natural NK cell cytotoxicity, while P815, a murine mastocytoma cell line $\mathrm{Fc} \gamma \mathrm{R}^{+}$, was the target for reverse ADCC. As effectors, we used the polyclonal NK cell cultures described above [12]. NK cell cyto- 
A
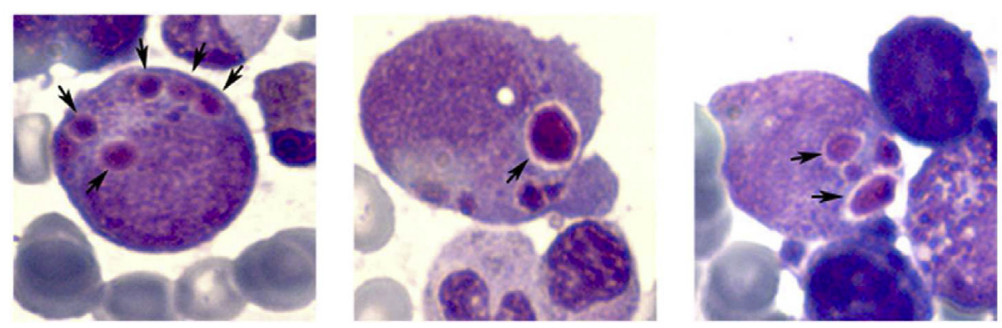

B
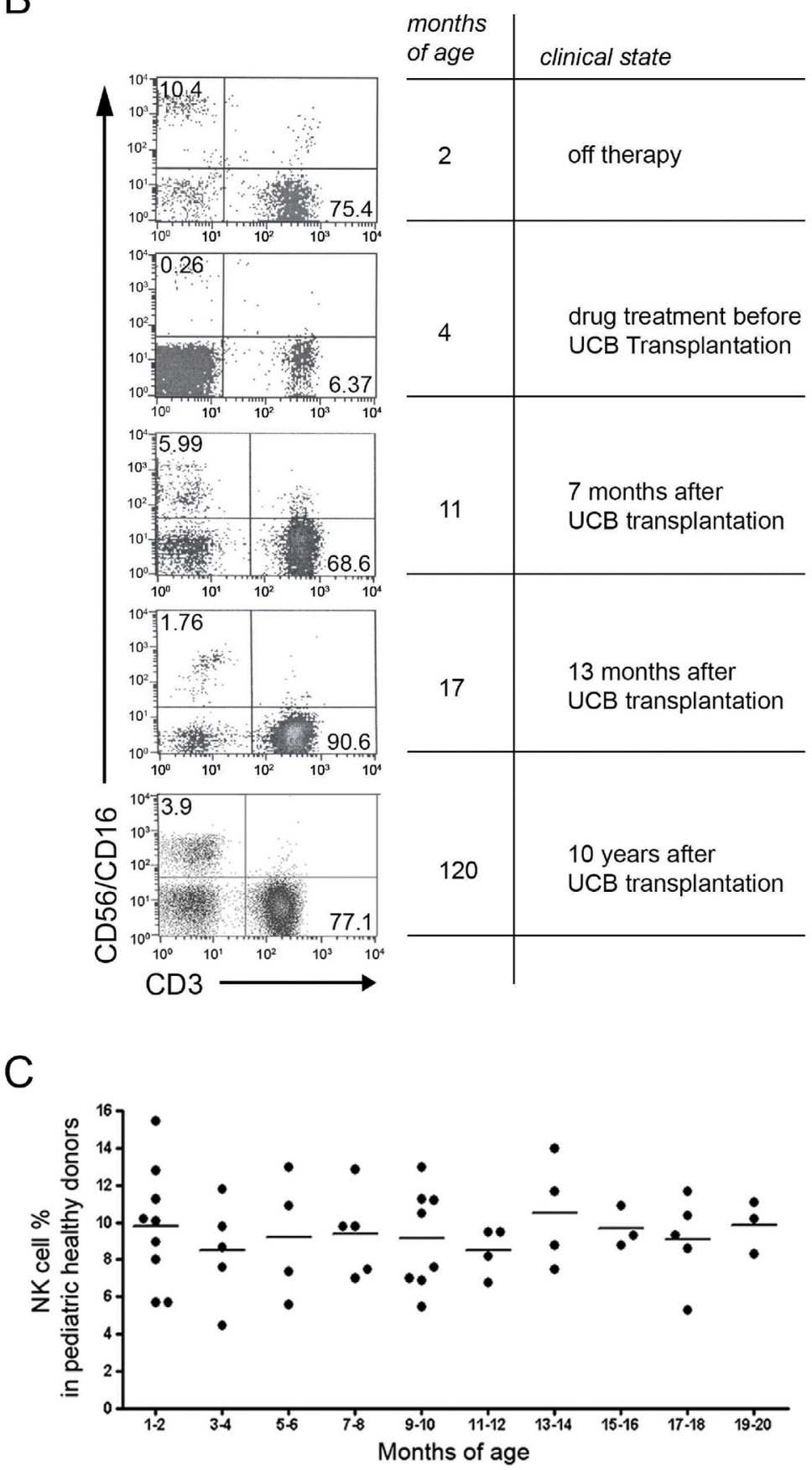

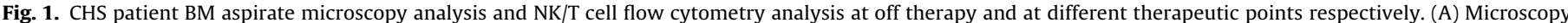

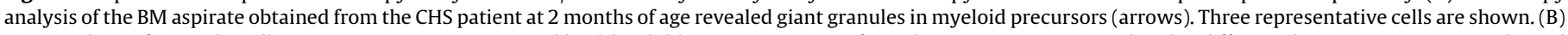

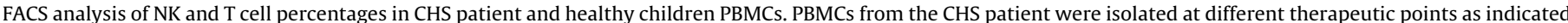

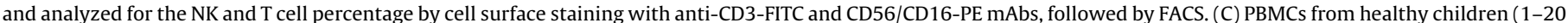
months of age) were used as controls. Black circles: NK cell percentage in each donor; horizontal bars: mean values in each group. 
toxicity was evaluated using the chromium $\mathrm{Cr}-51\left({ }^{51} \mathrm{Cr}\right)$ release assay.

\subsection{Evaluation of CHS NK cell/target cell binding formation, F-actin and perforin granule accumulation}

In vitro cultured $\mathrm{NK}$ cells $\left(3 \times 10^{6} / \mathrm{ml}\right)$, were mixed with equal volumes of K562 target cell suspensions (E/T ratio 5:1) and incubated in the dark for $15 \mathrm{~min}$ at $37^{\circ} \mathrm{C}$. After incubation, the pellet was gently resuspended and spun onto ice-cold poly-L-lysine-coated glass slides, fixed in $4 \%$ paraformaldehyde, permeabilized for $10 \mathrm{~min}$ at room temperature using FACS permeabilizing solution (BD Biosciences) and stained with FITC-conjugated anti-perforin $\mathrm{mAb}$ and rhodamine phalloidin. Granule polarization was assessed in NK cells forming conjugates with targets using a fluorescence microscope and the images were processed with Photoshop CS6 (Adobe, San Jose, CA).

\subsection{ELISA}

Enzyme-linked immunosorbent assays (ELISA) for IFN- $\gamma$ and TNF- $\alpha$ were from R\&D Systems. Cell-culture supernatants were analyzed without prior dilution, and the assays were performed according to manufacturer's instructions.

\subsection{Statistical analysis}

Statistical analysis was performed using a two-tailed Student's $t$-test. A value of $\mathrm{p}<0.05$ was considered statistically significant.

\section{Results}

\subsection{Analysis of NK cell percentages in the CHS patient before and after UCB transplantation}

Our patient was diagnosed as suffering from CHS on the basis of disease-specific giant granules present in cells of his BM aspirate (Fig. 1A), in addition to other typical signs of this syndrome described in methods.

PBMCs were isolated from CHS patient at different treatment points, or from pediatric healthy donors, and analyzed for NK and T cell percentages by CD56, CD16 and CD3 mAbs staining, followed by FACS analysis (Fig. 1B-C). The patient presented normal NK and T cell percentages when he was "off therapy" (before the antiblastic therapy), with approximately $10 \%$ of NK cells, a value similar to what observed in healthy children of 1-2 months of age (Fig. 1C and [14]). The percentages of both NK and T cells were strongly affected upon "drug treatment" (antiblastic therapy with etoposide, MTX and dexamethasone), a conditioning regimen for UCB-T (Fig. 1B). Engraftment was successful, since analysis of chimerism performed after the transplant, also on single leukocyte subsets as T, NK, B cells and monocytes, revealed $100 \%$ of engraftment (data not shown). Analysis of the percentage of NK cells in the peripheral blood of the patient at seven months, thirteen months and 10 years after UCB-T, showed that they were restored to almost normal levels compared to healthy children [14] (Fig. 1B-C and data not shown). By contrast, analysis of $T$ cells at the same treatment points indicated that there was a complete recovery of this lymphocyte subset (Fig. 1B).

\subsection{Analysis of NK cell-mediated cytotoxicity in the CHS patient before and after UCB transplantation and effect of in vitro IL-2 treatment}

To evaluate NK cell effector functions, NK cells derived from the patient or from healthy donors were cultured in vitro and then assayed for natural cytotoxicity and reverse ADCC [13], as well as for their ability to form NK-target cell contacts and to produce cytokines. The culture system results in the preferential proliferation of NK cells that, after 10-14 days, are in a state of low activation and resemble resting rather than activated cells [13]. In vitro cultured CHS NK cells showed a normal ability to proliferate in response to feeder cells, as demonstrated by the percentage of $\mathrm{CD}^{-} / \mathrm{CD}^{2} 6^{+} / \mathrm{CD} 16^{+}$cells [13] (Suppl. Fig. 1 ). Moreover, expanded CHS NK cells expressed normal levels of several receptors, including CD16, NKG2D, 2B4, NKp44, CD94/NKG2A, LFA-1/integrin beta2, HLA-DR, FAS, FASL and an increased expression of NKG2D and HLADR in response to IL-2 (data not shown).

In relation to the cytotoxic potential, we observed that in vitro expanded NK cells obtained from the patient at the time of hospitalization ("off therapy"), had a strong impairment of both natural cytotoxic activity and reverse-ADCC, compared to healthy donors (Fig. 2A-B) [15]. However, when NK cells derived from the patient were treated with IL-2 $(250 \mathrm{IU} / \mathrm{mL})$ for $18 \mathrm{~h}$, cytotoxicity levels were comparable to those of IL-2-treated cultured NK cells derived from healthy controls (Fig. 2A-B). An impairment of NK cell-mediated cytotoxicity was observed also at later time points, when the patient underwent a pre-transplant conditioning regimen and then after UCB-T, but also in these cases, IL-2 was able to correct the defect (Fig. 2C-H). The positive effect of IL-2 was observed also at lower doses (from 10 to $125 \mathrm{IU} / \mathrm{ml}$, for $48 \mathrm{~h}$ ) (data not shown). At 10 years after transplantation, the patient showed an almost normal cytotoxic ability of NK cells which was further enhanced by IL-2 (Fig. 2I-L). These results show that the defects in NK cell lysis associated with CHS disease could be restored upon IL-2 treatment, and suggest that UCB-T represents an acceptable curative treatment for these patients.

\subsection{IL-2 increases perforin-containing granules into contact sites of CHS-derived NK cells with target cells}

To better investigate the mechanisms responsible for the increased cytotoxic ability of CHS NK cells after IL-2 treatment, we analyzed the capacity of patient-derived NK cells to form the immunological synapse (IS), by measuring the ability to bind target cells and to mediate natural cytotoxicity through perforin production (Fig. 3). NK/target cell conjugate formation was investigated by performing binding tests with NK cells obtained from the patient when he was "off therapy", or from healthy donors as controls, and with K562 used as targets. Cells were stained with a specific probe to survey the amount of actin polymerization at the IS and with a specific $\mathrm{mAb}$ against perforin, and the contact sites were analyzed by fluorescence microscopy (Fig. 3).

Compared to a healthy donor, CHS NK cells showed bigger perforin granules (Fig. 3), likely derived from fused and enlarged secretory lysosomes, as reported before for T lymphocytes [16,17]. The granules were localized far from the NK-target cell binding site; however, IL-2 treatment resulted in the appearance of more numerous and smaller granules and in their positioning in the contact area, with a pattern similar to the one observed with the healthy control. These observations paralleled the increased cytotoxic potential observed upon IL-2-treatment (Fig. 2). Finally, the number of effector-target cell bindings and the accumulation of $\mathrm{F}$ actin observed in the contact area of CHS NK cells did not show anomalies, being similar to healthy controls (Fig. 3 and data not shown).

Thus, the observed capability of IL-2 to restore CHS NK cellmediated cytotoxicity may derive from the recovery to a normal perforin-containing granule distribution and localization, as well as granule exocytosis, upon NK-target cell contact, and this may have an important role in regaining NK cell functions in CHS patients. 


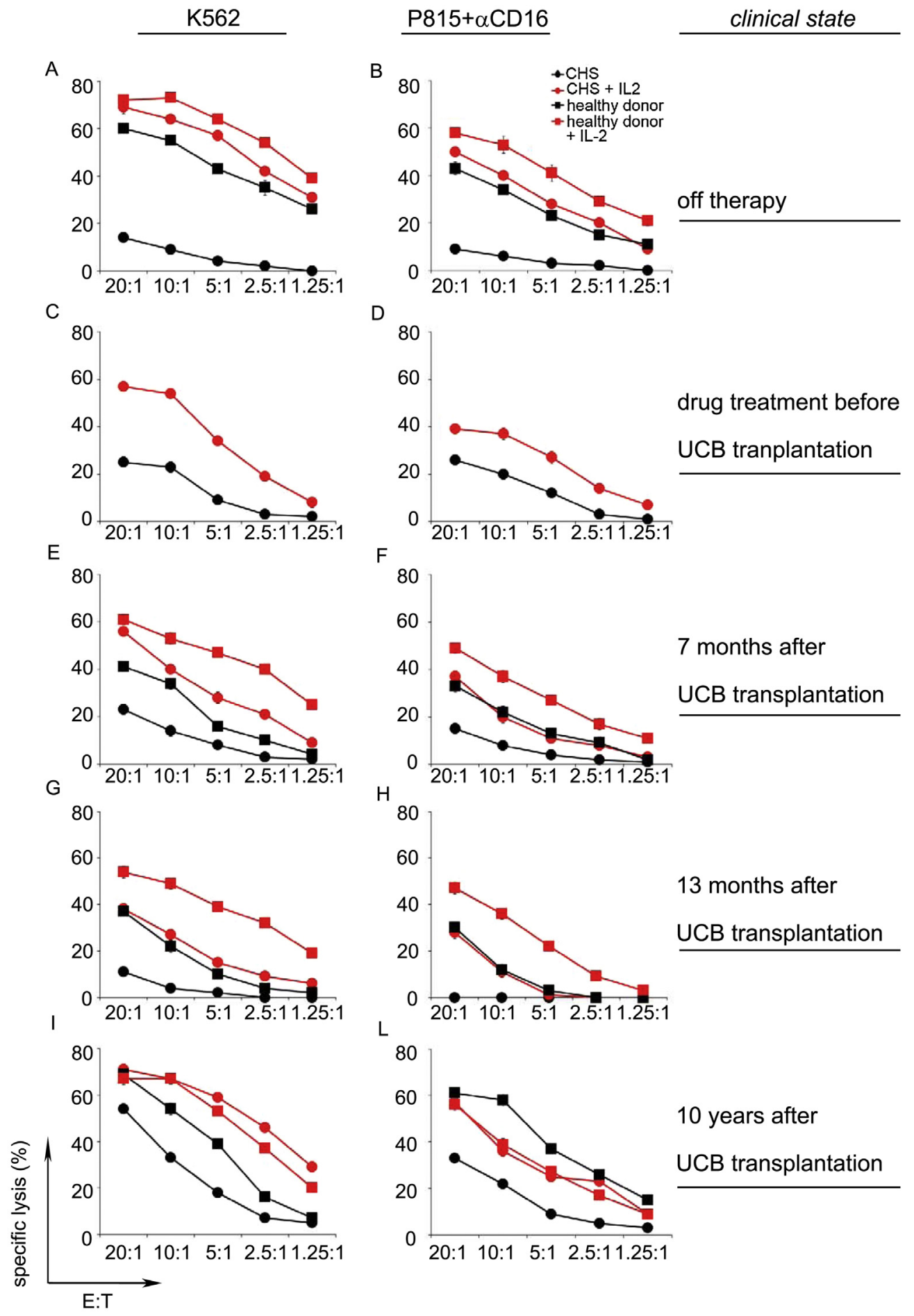

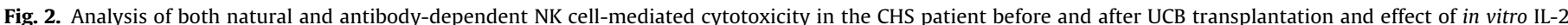

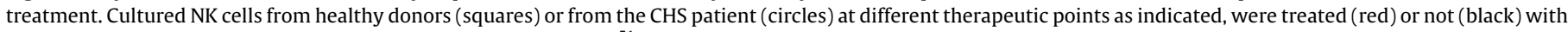

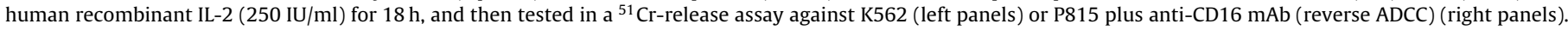
(For interpretation of the references to colour in this figure legend, the reader is referred to the web version of this article.)

\subsection{Analysis of CHS NK cell cytokine production and effect of in vitro IL-2 treatment}

We then analyzed the ability of in vitro expanded CHS-derived NK cells, obtained from the patient when he was "off therapy", to produce IFN- $\gamma$ and TNF- $\alpha$ cytokines (Fig. 4A-B). We observed a strong impairment of IFN- $\gamma$ production by CHS NK cells, as eval- uated both by intracellular staining (data not shown) and ELISA (Fig. 4A). Similarly to what we observed in cytotoxicity assays, IL-2 treatment was able to correct the defect (Fig. 4A). A similar effect was observed also on the production of TNF- $\alpha$ (Fig. 4B).

Altogether, our results indicate that the altered NK cell cytotoxic activity, lytic granule pattern, and cytokine production we observed in the CHS patient, could be reversed by IL-2 treatment. 
healthy donor
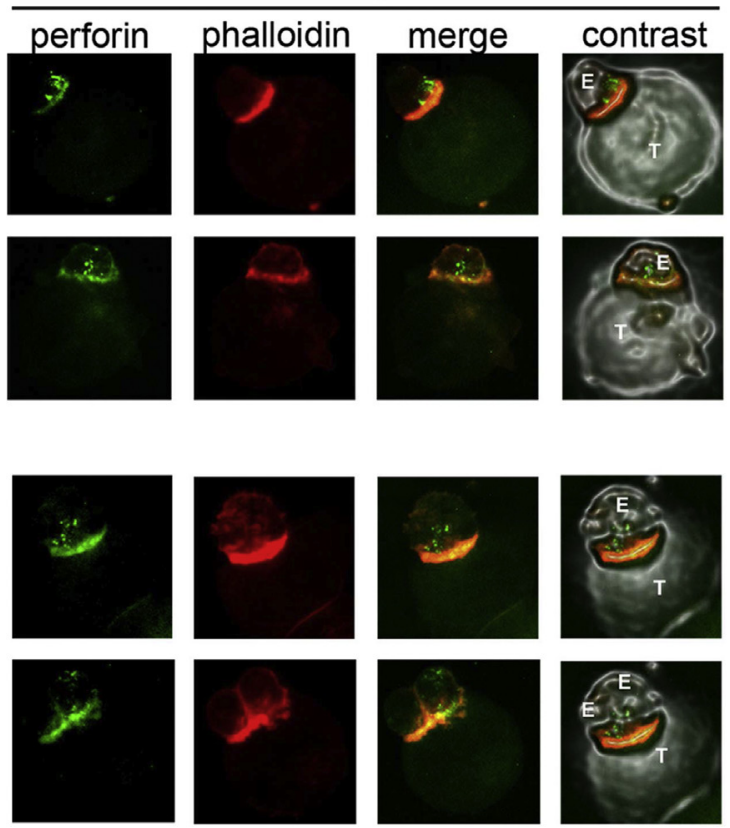
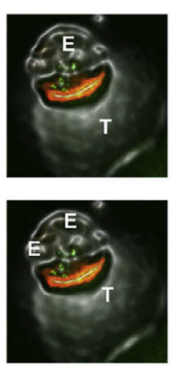

CHS patient
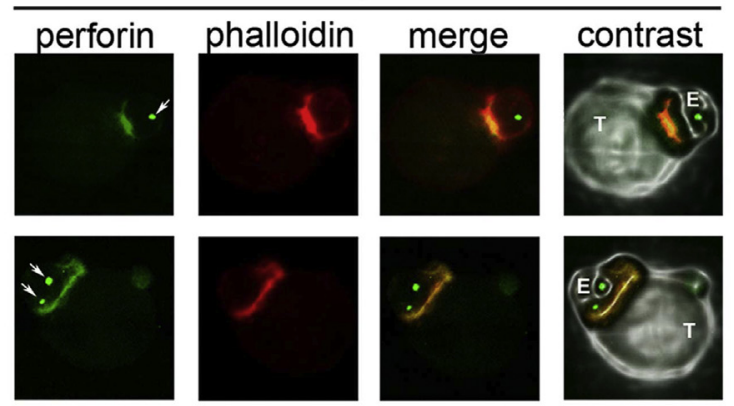

medium
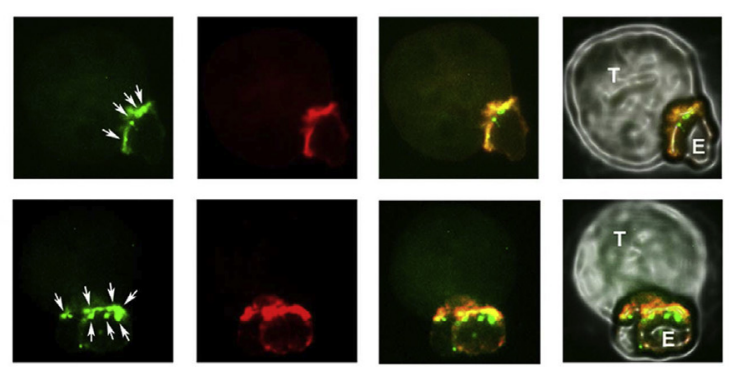

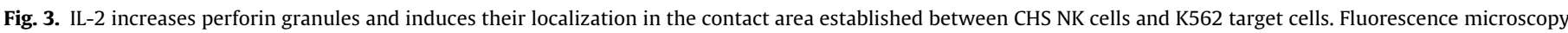

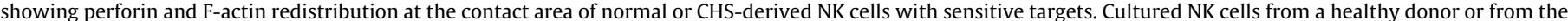

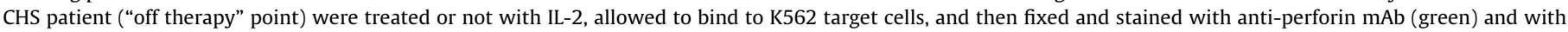

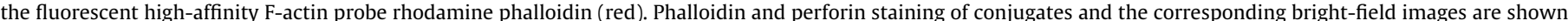

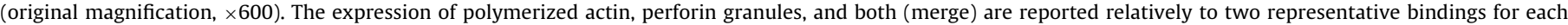

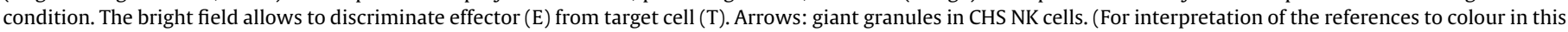
figure legend, the reader is referred to the web version of this article.)

\section{Discussion}

Chédiak-Higashi syndrome is a pathological condition affecting several tissues and organs. Both innate and adaptive immune responses are severely compromised, and this renders leukocytes unable to effectively fight off pathogens, such as viruses and bacteria. For this reason, most of the patients affected by CHS have recurrent and persistent infections, starting in infancy or in early childhood. These infections tend to be life-threatening, with few patients living to adulthood [1]. Treatment of CHS patients is mainly aimed at enforcing the severely impaired immune system, in order to confer the ability to respond against bacterial and viral insults. At present, the most significant clinical intervention consists in hematopoietic stem cell (HSC) transplantation, mainly of BM origin $[10,18-21]$. However, the use of UCB-T in patients with CHS has been recently reported [11].

More recently, other authors reported outcomes after HSC transplantation of a significant cohort of CHS patients (35 children) [22]. With a median follow-up of 6.5 years, the 5-year probability of overall survival was only $62 \%$. Due to the severity of this disease, almost all patients at the moment of HSC transplantation presented an active AP symptomatology that strongly compromised the outcome, leading to a high mortality in both early and late phases post-HSC transplantation. Remission, which not always corresponded to survival, was obtained only in those cases of disease-free condition at transplantation, performed with HSC from either HLA-matched sibling donors or unrelated donors.

In this context, we analyzed the functional efficacy of UCB-T on NK cell effector functions, in an in vitro study performed on NK cells derived from a CHS patient before and after UCB-T. Firstly, we investigated NK cell cytotoxic function, both natural and antibodydependent and, as reported also by other authors [4-7,23], they both resulted severely impaired. However, we observed that treat- ment of CHS-derived NK cell cultures with IL-2 could completely restore their lytic potential. This is in line with previous reports showing that cytotoxicity mediated by PBLs or NK cells obtained from CHS patients could be restored by type I IFN, while cytotoxicity of total PBMCs or of virally-transformed T cell lines could be restored by IL-2 [4,23-25]. However, the specific restoration of CHS NK cell defects by IL-2 has never been reported before. There is only one report describing unsuccessful NK cell degranulation upon IL-2 treatment, but the discrepancy between our study and the previous one could derive from differences in the amount of IL-2, cell culture conditions, and/or cytotoxicity assay [26].

By fluorescence microscopy analysis, we also observed for the first time how IL-2 was able to restore natural NK cell-mediated cytotoxicity. Compared to untreated CHS NK cells (our study and $[16,17,27])$, perforin-containing granules appeared smaller and redistributed upon IL-2 treatment, with an orientation towards the IS established with sensitive targets.

The analysis of samples obtained from our CHS patient at different treatment points, before and after the successful UCB-T, allowed us to observe a partial restoration of NK cell cytotoxic ability, which was particularly evident several years after the transplant. The absence of a full recovery at earlier times could be related to the fact that the infusion of allogeneic HSC does not ensure in CHS patients the functional reconstitution of the so called "environmental niche" [28-30].

It is known, in fact, that during lymphocyte development, the BM is the site where several events of NK cell development occur, which can influence and/or determine the final functional fate of NK cell precursors [28]. These events include the generation of NK cell precursors from HSC, the acquisition of cell surface receptors involved in target-cell recognition, the NK cell education, and the emergence of differentiated NK cells with fully functional competence [29]. 
A

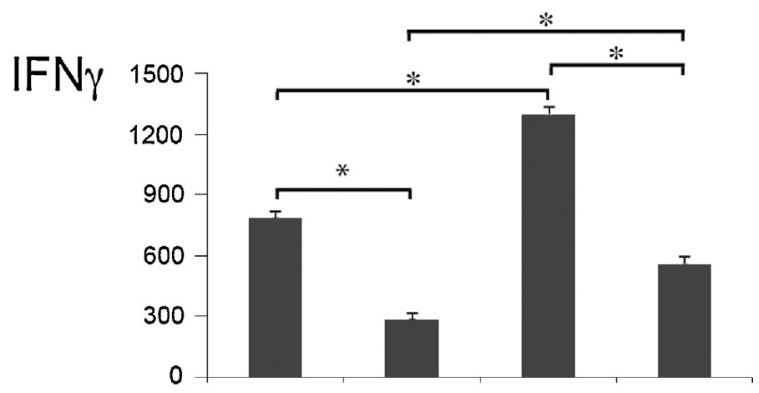

B

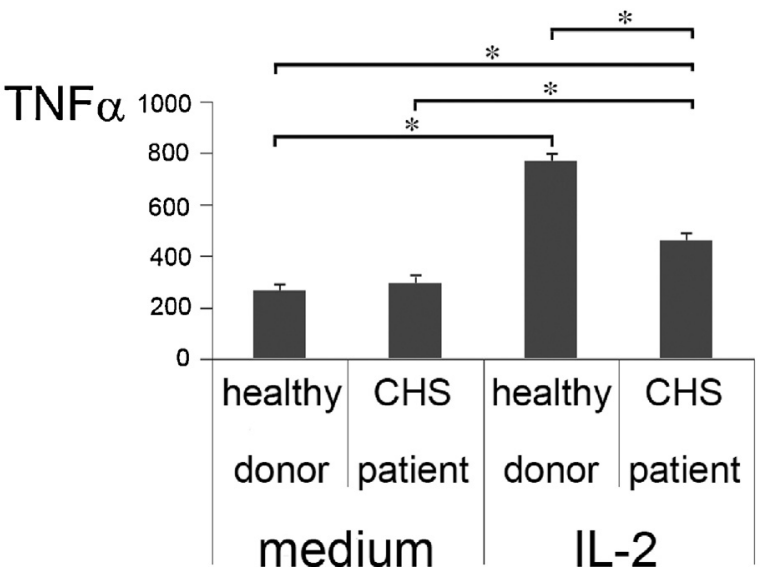

Fig. 4. IL-2 increases IFN- $\gamma$ and TNF- $\alpha$ production in CHS-derived NK cells. Cultured NK cells from a healthy donor or from the CHS patient (at the "off therapy" point), were treated or not with human recombinant IL-2 $(250 \mathrm{IU} / \mathrm{ml})$ for $18 \mathrm{~h}$, and then tested for the production of cytokines. Supernatants were collected and tested for the presence of A) IFN- $\gamma$ and B) TNF- $\alpha$. ${ }^{*} p<0.05$ with Student's $t$-test.

Another aspect that might influence the reconstitution of NK cell effector functions comes from the evidence that UCB-derived NK cells have less in vitro killing ability, which can be augmented by cytokine stimulation, including IL-2, with a final cytotoxicity not different from similarly treated NK cells from healthy donors [31-33]. Thus, the delay in the full recovery of NK killing ability could be the result both of the genetic defect and of the source of HSC.

An important point of NK cell biology that has emerged in the recent past is that these cells are not only crucial effectors of innate immunity, but they also play an important role in determining the outcome of adaptive immune responses [34]. In fact, NK cells are able to promptly produce cytokines (e.g., TNF- $\alpha$ and IFN- $\gamma$ ) and chemokines that help to generate and sustain an inflammatory environment [35]. Because of the functional importance of TNF- $\alpha$ and IFN- $\gamma$, we explored the ability of CHS-derived NK cells to produce these cytokines. As comparably reported also by other authors [3], we observed that CHS NK cells showed an almost normal TNF- $\alpha$ production, but they were strongly impaired in producing IFN- $\gamma$, by elucidating a more complex picture linked to the functional impairment of NK cells in this syndrome. However, upon in vitro IL-2 treatment, there was a partial restoration in the production of both cytokines, though at lower levels compared to healthy donors.

In conclusion, the observation of NK cell cytotoxicity restoration mediated by in vitro IL-2 treatment before and after UCB-T, suggests that this cytokine could be adopted in the clinical therapy of CHS, though this choice should be limited in time and doses [36] considering the toxic side effects of this cytokine. Our results also indicate that treatment of these patients with IL-2 could activate not only the innate immune functions mediated by NK cells, but also a $\mathrm{T}$ cell-dependent response due to the immunomodulatory role of
NK cells. For all these reasons, it is plausible to hypothesize a new scenario with the clinical adoption of IL-2 in the treatment of CHS patients, in order to improve immune responses and more rapidly obtain a successful transplant. Of note, this is a treatment already envisaged in other primary immunodeficiencies, including SCID, Wiskott-Aldrich syndrome, NEMO, CVID and Ataxia Telangiectasia $[12,37]$.

\section{Competing interests}

The authors declare that they have no competing interests.

\section{Authors' contributions}

LCif and CCer were the principal investigators, performed the laboratory work and take primary responsibility for the paper; RMP, IR, MC, LCur recruited and treated the patient by following his clinical state; AA, SP, CCan, GP and PR diagnosed the syndrome and provided the patient samples to the authors involved in the experimental work; LCif, CCer, AZ, AG, AS coordinated the research; LCif, CCer, AG and AS wrote the paper. All authors read and approved the final manuscript.

\section{Funding}

This work was supported by grants from the Italian Association for Cancer Research (AIRC Investigator Grant and Special Project “ $5 \times 1000$ " Grant \#9962), the Italian Ministry of University and Research (MIUR) (PRIN) and the Center of Excellence (BEMM) to Angela Santoni; the Italian Ministry of Health (Rome, Italy) Grant GR-2011-02352151 to Loredana Cifaldi; the Pasteur Institute - Cenci-Bolognetti Foundation and the "Sapienza" University of Rome to Cristina Cerboni.

\section{Acknowledgements}

The authors would like to thank Prof. Franco Locatelli for critically reading this manuscript and for stimulating and fruitful discussions. The authors are grateful to Dr. Geneviève de Saint Basile, Hôpital Necker-Enfants Malades, INSERM U429 Paris, for the genetic analysis of the patient.

\section{Appendix A. Supplementary data}

Supplementary data associated with this article can be found, in the online version, at http://dx.doi.org/10.1016/j.imlet.2016.10. 009.

\section{References}

[1] W. Introne, R.E. Boissy, W.A. Gahl, Clinical, molecular, and cell biological aspects of Chediak-Higashi syndrome, Mol. Genet. Metab. 68 (1999) 283-303.

[2] S. Certain, F. Barrat, E. Pastural, F. Le Deist, J. Goyo-Rivas, N. Jabado, et al., Protein truncation test of LYST reveals heterogenous mutations in patients with Chediak-Higashi syndrome, Blood 95 (2000) 979-983.

[3] A. Gil-Krzewska, S.M. Wood, Y. Murakami, V. Nguyen, S.C. Cher Chiang A.R. Cullinane, et al., Chediak-Higashi syndrome: lysosomal trafficking regulator domains regulate exocytosis of lytic granules but not cytokine secretion by natural killer cells, J. Allergy Clin. Immunol. (2015).

[4] T. Haliotis, J. Roder, M. Klein, J. Ortaldo, A.S. Fauci, R.B. Herberman, Chediak-Higashi gene in humans I. Impairment of natural-killer function, J. Exp. Med. 151 (1980) 1039-1048.

[5] M. Klein, J. Roder, T. Haliotis, S. Korec, J.R. Jett, R.B. Herberman, et al., Chediak-Higashi gene in humans: II. The selectivity of the defect in natural-killer and antibody-dependent cell-mediated cytotoxicity function, J. Exp. Med. 151 (1980) 1049-1058.

[6] P. Katz, A.M. Zaytoun, A.S. Fauci, Deficiency of active natural killer cells in the Chediak-Higashi syndrome: localization of the defect using a single cell cytotoxicity assay, J. Clin. Invest. 69 (1982) 1231-1238. 
[7] S.R. Targan, R. Oseas, The lazy NK cells of Chediak-Higashi syndrome, J. Immunol. 130 (1983) 2671-2674.

[8] O. Yegin, O. Sanal, O. Yeralan, A. Gurgey, A.I. Berkel, Defective lymphocyte locomotion in Chediak-Higashi syndrome, Am. J. Dis. Child. 137 (1983) $771-773$.

[9] M. Bejaoui, F. Veber, D. Girault, C. Gaud, S. Blanche, C. Griscelli, et al., [The accelerated phase of Chediak-Higashi syndrome], Arch. Fr. Pediatr. 46 (1989) 733-736.

[10] E. Haddad, F. Le Deist, S. Blanche, M. Benkerrou, P. Rohrlich, E. Vilmer, et al., Treatment of Chediak-Higashi syndrome by allogenic bone marrow transplantation: report of 10 cases, Blood 85 (1995) 3328-3333.

[11] R. Rihani, M. Barbar, N. Faqih, H. Halalsheh, A.A. Hussein, A.H. Al-Zaben, et al., Unrelated cord blood transplantation can restore hematologic and immunologic functions in patients with Chediak-Higashi syndrome, Pediatr. Transplant. 16 (2012) E99-E105

[12] A. Gismondi, L. Cifaldi, C. Mazza, S. Giliani, S. Parolini, S. Morrone, et al., Impaired natural and CD16-mediated NK cell cytotoxicity in patients with WAS and XLT: ability of IL-2 to correct NK cell functional defect, Blood 104 (2004) 436-443.

[13] B. Perussia, C. Ramoni, I. Anegon, M.C. Cuturi, J. Faust, G. Trinchieri, Preferential proliferation of natural killer cells among peripheral blood mononuclear cells cocultured with B lymphoblastoid cell lines, Nat. Immun. Cell Growth Regul. 6 (1987) 171-188.

[14] W.M. Comans-Bitter, R. de Groot, R. van den Beemd, H.J. Neijens, W.C. Hop, K. Groeneveld, et al., Immunophenotyping of blood lymphocytes in childhood: reference values for lymphocyte subpopulations, J. Pediatr. 130 (1997) 388-393.

[15] A. Yabuhara, H. Kawai, A. Komiyama, Development of natural killer cytotoxicity during childhood: marked increases in number of natural killer cells with adequate cytotoxic abilities during infancy to early childhood, Pediatr. Res. 28 (1990) 316-322.

[16] S.L. Shiflett, J. Kaplan, Ward D.M. Chediak-Higashi Syndrome, a rare disorder of lysosomes and lysosome related organelles, Pigment Cell Res. 15 (2002) 251-257.

[17] D.M. Ward, S.L. Shiflett, J. Kaplan, Chediak-Higashi syndrome: a clinical and molecular view of a rare lysosomal storage disorder, Curr. Mol. Med. 2 (2002) 469-477.

[18] M. Mottonen, M. Lanning, U.M. Saarinen, Allogeneic bone marrow transplantation in Chediak-Higashi syndrome, Pediatr. Hematol. Oncol. 12 (1995) 55-59.

[19] M. Miano, F. Porta, F. Locatelli, R. Miniero, G. La Nasa, P. Di Bartolomeo, et al. Unrelated donor marrow transplantation for inborn errors, Bone Marrow Transplant. 21 (Suppl 2) (1998) S37-41.

[20] S. Yamazaki, H. Takahashi, H. Fujii, T. Miyamae, M. Mori, K. Fujioka, et al., Split chimerism after allogeneic bone marrow transplantation in Chediak-Higashi syndrome, Bone Marrow Transplant. 31 (2003) 137-140.
[21] I.H. Bartelink, S.V. Belitser, C.A. Knibbe, M. Danhof, A.J. de Pagter, T.C. Egberts, et al., Immune reconstitution kinetics as an early predictor for mortality using various hematopoietic stem cell sources in children, Biol. Blood Marrow Transplant. 19 (2012) 305-313.

[22] M. Eapen, B.R. Logan, D.L. Confer, M. Haagenson, J.E. Wagner, D.J. Weisdorf, et al., Peripheral blood grafts from unrelated donors are associated with increased acute and chronic graft-versus-host disease without improved survival, Biol. Blood Marrow Transplant. 13 (2007) 1461-1468.

[23] T. Nakazawa, K. Agematsu, K. Yasui, T. Onodera, R. Inoue, H. Kaneko, et al., Cytolytic mechanisms involved in non-MHC-restricted cytotoxicity in Chediak-Higashi syndrome, Clin. Exp. Immunol. 118 (1999) 108-114.

[24] J.M. Martin-Fernandez, J.A. Cabanillas, M. Rivero-Carmena, E. Lacasa, J. Pardo, A. Anel, et al., Herpesvirus saimiri-transformed CD8+ T cells as a tool to study Chediak-Higashi syndrome cytolytic lymphocytes, J. Leukoc. Biol. 77 (2005) 661-668.

[25] R.F. Holcombe, Interleukin-2-induced cytotoxicity of Chediak-Higashi lymphocytes, Acta Haematol. 87 (1992) 45-48.

[26] Y.T. Bryceson, D. Pende, A. Maul-Pavicic, K.C. Gilmour, H. Ufheil, T. Vraetz, et al., A prospective evaluation of degranulation assays in the rapid diagnosis of familial hemophagocytic syndromes, Blood 119 (2012) 2754-2763.

[27] O. Higashi, Congenital gigantism of peroxidase granules; the first case ever reported of qualitative abnormity of peroxidase, Tohoku J. Exp. Med. 59 (1954) 315-332.

[28] N.D. Huntington, C.A. Vosshenrich, J.P. Di Santo, Developmental pathways that generate natural-killer-cell diversity in mice and humans, Nat. Rev. Immunol. 7 (2007) 703-714

[29] F. Colucci, M.A. Caligiuri, J.P. Di Santo, What does it take to make a natural killer? Nat. Rev. Immunol. 3 (2003) 413-425.

[30] J.P. Di Santo, Natural killer cells: diversity in search of a niche, Nat. Immunol. 9 (2008) 473-475.

[31] R. Danby, V. Rocha, Improving engraftment and immune reconstitution in umbilical cord blood transplantation, Front. Immunol. 5 (2014) 68

[32] M.R. Verneris, J.S. Miller, The phenotypic and functional characteristics of umbilical cord blood and peripheral blood natural killer cells, Br. J. Haematol. 147 (2009) 185-191.

[33] M. Luevano, M. Daryouzeh, R. Alnabhan, S. Querol, S. Khakoo, A. Madrigal, et al., The unique profile of cord blood natural killer cells balances incomplete maturation and effective killing function upon activation, Hum. Immunol. 73 (2012) 248-257.

[34] A. Zingoni, M. Ardolino, A. Santoni, C. Cerboni, NKG2D and DNAM-1 activating receptors and their ligands in NK-T cell interactions: role in the NK cell-mediated negative regulation of T cell responses, Front. Immunol. 3 (2013) 408.

[35] G. Trinchieri, Biology of natural killer cells, Adv. Immunol. 47 (1989) 187-376.

[36] A delicate balance: tweaking IL-2 immunotherapy. Nature medicine 18 (2012) 208-209.

[37] S. Roy-Ghanta, J.S. Orange, Use of cytokine therapy in primary immunodeficiency, Clin. Rev. Allergy Immunol. 38 (2010) 39-53. 